\title{
Mechanisms of spinal cord stimulation in ischemia
}

\author{
Oren Sagher, M.D., and Dah-Luen Huang, M.S. \\ Department of Neurosurgery, University of Michigan, Ann Arbor, Michigan
}

\begin{abstract}
Object. The goal of this study was to assess the duration of neuroprotection after SCS. Nearly 40 years after the first description of spinal cord stimulation (SCS), the mechanisms underlying its physiological effects remain unclear. It is known that SCS affects activity in the nervous system on a broad scale. Local neurohumoral changes within the dorsal horn of the spinal cord have been described, as have changes in cortical activation in a number of brain regions. Spinal cord stimulation has even been found to have profound effects on sympathetic vascular tone, a discovery that has led to its use in ameliorating blood flow in the limbs, heart, and brain.

Methods. In an effort to delineate the limits of neuroprotection offered by SCS, the authors have studied its use in an experimental model of permanent middle cerebral artery (MCA) occlusion in rats. The investigators applied SCS in a delayed fashion 3, 6, or 9 hours after MCA occlusion. The results are reported and mechanisms underlying the physiological effects of SCS are reviewed, with particular attention being paid to the effect of SCS on cerebral blood flow in the setting of cerebral ischemia.

Conclusions. The authors found that SCS applied as late as 6 hours postischemia significantly reduces stroke volumes, whereas SCS applied 9 hours after ischemia fails to reduce stroke injury.
\end{abstract}

\section{KEY WORDS • pain • stroke • cerebral blood flow • electrical stimulation • spinal cord stimulation $\bullet$ rat}

$\mathrm{T}$ HE development of SCS as a therapeutic modality for the management of chronic pain followed the initial description of the gate control theory of nociception in $1965 . .^{22}$ Shealy and colleagues ${ }^{28}$ reasoned that the electrical "gates" within the dorsal aspect of the spinal cord that were postulated by Melzack and Wall could be artificially stimulated by electrical current applied externally. The technology of SCS has evolved over the succeeding three decades, but the underlying concept remains unchanged. Modern SCS units consist of an electrical lead positioned in the epidural space overlying the dorsal spinal cord and a pulse generator that delivers a high-frequency, square-wave current. When the spinal cord is stimulated, patients describe a vibratory sensation in their extremities. This sensation is associated with a significant reduction of pain in the "stimulated" limbs. Since its introduction in 1967, SCS has been widely used for the treatment of chronic pain. ${ }^{24,30}$ This treatment is believed to alter neuronal inputs and synaptic activity within the dorsal horn of the spinal cord, thereby reducing central transmission of pain. Electrical conduction in the dorsal columns of the spinal cord has been thought to be modified by SCS, although this concept has been challenged. ${ }^{4}$ The mechanism of action of SCS is the subject of heated debate,,$^{30}$ although its efficacy in the alleviation of chronic pain is largely accepted.

Placement of the SCS leads varies widely according to

\footnotetext{
Abbreviations used in this paper: $\mathrm{CBF}=$ cerebral blood flow; MCA = middle cerebral artery; SCS = spinal cord stimulation; TTC $=2,3,5$-triphenyltetrazolium chloride.
}

clinical indications and patient anatomy. The leads are routinely placed in the cervical and in the thoracolumbar spine for the treatment of arm and leg pain, respectively. Optimal lead location for the treatment of pain in various regions has been the subject of some investigations. ${ }^{2} \mathrm{Nev}-$ ertheless, the location of the SCS lead is usually determined by clinical benefit, and may range from the top of the cervical spine to the conus medullaris at its lower end. Mathematical modeling of SCS has also been done in an effort to predict its effects on the spinal cord. Finite-element modeling work performed by Holsheimer and colleagues ${ }^{8-10}$ has enabled accurate prediction of electrical contact geometries, providing optimal penetration of the dorsal aspect of the spinal cord.

\section{Use of SCS in Peripheral and Myocardial Ischemia}

A serendipitous discovery by Cook and others ${ }^{5}$ while using SCS to treat ischemic limb pain in 1976 led them to postulate a direct effect on peripheral vascular tone. In this landmark paper, a patient is described in whom painful ischemic ulcers were observed in the lower extremities. Following the successful application of SCS, the patient's pain was alleviated. In addition, the perfusion to the lower extremities improved noticeably and the patient's ischemic ulcers began to heal. When SCS was stopped, the pain and (particularly noteworthy) the ulcers reappeared. Reestablishment of SCS once again resulted in improvement in the symptomatic peripheral ischemia. Since this initial observation was reported, the effects of SCS on vascular tone in the peripheral circulation have been studied extensively in the laboratory. ${ }^{16-18,23}$ Blood 
flow in the rat hindpaw has been shown to rise dramatically in response to stimulation of the lumbar spinal cord. ${ }^{16}$ It has also been reported that both chemical and surgical sympathectomy blunted the vascular response to SCS. ${ }^{16,17}$ Finally, SCS-induced blood flow changes were noted to occur despite transection of the rostral spinal cord or the dorsal nerve roots. Based on these lines of experimental evidence, researchers have suggested that SCS reduces peripheral sympathetic vascular tone, thereby augmenting blood flow in the limbs. ${ }^{18}$

The therapeutic efficacy of SCS in the management of ischemic limb pain is now accepted. ${ }^{14,15,33}$ It has also been suggested that SCS may reduce ischemic tissue injury in patients with peripheral vascular disease, although the data supporting this conclusion are mixed. ${ }^{14,15}$ Currently, application of SCS for symptomatic peripheral ischemia constitutes one of the most common indications for this treatment in Europe.

In addition to its use in peripheral vascular disease, SCS has been reported to be successful in reducing pain from myocardial ischemia. In a multicenter, randomized, prospective trial ${ }^{19}$ it has been demonstrated that, far from masking incipient myocardial infarction, SCS is accompanied by improvement in coronary perfusion and inotropic performance. Furthermore, analysis of the data from this trial found that both coronary artery bypass grafting and SCS conferred similar protection from angina episodes and myocardial infarction over a 5-year period. ${ }^{6}$ Equivalence has also been found between percutaneous revascularization and SCS. ${ }^{20}$ When considered in aggregate, these observations indicate that SCS has a clinically significant vasodilatory effect in the peripheral vasculature and may even improve blood flow to ischemic tissue.

\section{Use of SCS in Cerebral Ischemia}

Because SCS has been shown to enhance peripheral vasodilation, it can also be considered for augmentation of cerebral perfusion. Changes in CBF related to SCS were the subject of an anecdotal report by Hosobuchi ${ }^{11}$ involving a small number of patients who underwent stimulation for treatment of chronic pain. Hosobuchi and others ${ }^{3,12,13}$ found that high cervical SCS increased CBF, although the extent of this augmentation and the underlying mechanisms have not been clearly defined. The possibility that CBF may be augmented with SCS has led to attempts to use stimulation in the treatment of cerebral ischemia. ${ }^{3}$ Several investigators have applied SCS in experimental models of cerebral ischemia and in physiological preparations designed to measure CBF. $3.7,13,21,35$ In goats and dogs, Garcia-March and colleagues ${ }^{7}$ found that electrical stimulation at the C-2 spinal segment increased CBF by 55\% when measured with laser Doppler flowmetry and by $35 \%$ when studied quantitatively by using iodoantipyrene autoradiography. Visocchi and coworkers ${ }^{35}$ showed that CBF in rabbits will be increased by cervical SCS but can be attenuated by concurrent stimulation of the sympathetic trunk. Using a cat model, Isono and colleagues ${ }^{13}$ found that CBF changes occurred only with SCS performed in the cervical spine. Moreover, these authors discovered that sectioning of the dorsal columns at the cervicomedullary junction abolished CBF changes. These findings have been corroborated independently in our laboratory by using a rat model of focal cerebral ischemia. ${ }^{25,26}$

The application of SCS also appears to result in a dramatic reduction of infarct volume in the setting of focal cerebral ischemia. ${ }^{27}$ The experimental evidence accumulated to this point indicates that SCS has the capacity to improve cerebral perfusion and reverse ischemic injury in the brain, perhaps by alterations in sympathetic tone as well as indirect activation of brainstem or cerebellar vasomotor centers.

A number of clinical reports describing the use of SCS in the treatment of patients with cerebral ischemia have been published. Small numbers of patients and a variety of CBF measurement methods have been described in these reports, and investigators have suggested that SCS does indeed augment CBF during ischemia. In 2000, Takanashi and Shinonaga ${ }^{31}$ published a report on a small series of patients who underwent SCS in the management of cerebral vasospasm following subarachnoid hemorrhage. These patients were studied before SCS was applied and 4 days after its use with xenon-inhalation computed tomography studies. The authors reported that regional CBF values were stable or slightly increased after the use of SCS, despite the fact that CBF normally decreases in patients over this time period. Moreover, there were no ad-

\section{TABLE 1}

Literature review of SCS data in patients with cerebral ischemia*

\begin{tabular}{|c|c|c|c|c|}
\hline Authors \& Year & No. of Patients & Disease Process & CBF Measure & Outcome \\
\hline Hosobuchi, 1991 & 3 & symptomatic cerebral ischemia & Xe-CT, SPECT & increased $\mathrm{CBF}$, extent not specified \\
\hline Visocchi et al., 1994 & 1 & stroke & TCD & $\begin{array}{l}43 \% \text { (contralat) to } 130 \% \text { (ipsilat) } \\
\text { increase in MCA flow velocity }\end{array}$ \\
\hline Broseta et al., 1994 & 10 & cerebral low-perfusion syndromes & SPECT & increased $\mathrm{CBF}$ in penumbral area \\
\hline $\begin{array}{l}\text { Takanashi \& } \\
\text { Shinonaga, } 2000\end{array}$ & 10 & cerebral vasospasm & $\mathrm{Xe}-\mathrm{CT}$ & $\begin{array}{l}\text { CBF in MCA distribution } \\
\text { increased } 20 \% \text { w/ SCS }\end{array}$ \\
\hline Visocchi et al., 2001 & 18 & stroke & $\begin{array}{l}\text { TCD, SPECT, } \\
\text { NIRS }\end{array}$ & $\begin{array}{l}\text { SPECT: CBF increase in } 9 \text { of } 12 \\
\text { patients; TCD: velocity increase } \\
\text { in } 4 \text { of } 11 \text { patients; NIRS: CBF } \\
\text { increase in } 1 \text { of } 1 \text { patients }\end{array}$ \\
\hline $\begin{array}{l}\text { Shinonaga \& } \\
\text { Takanashi, } 2001\end{array}$ & 12 & cerebral vasospasm & $\mathrm{Xe}-\mathrm{CT}$ & $\begin{array}{l}50 \% \text { of patients exhibited increase } \\
\text { in CBF w/ SCS }\end{array}$ \\
\hline
\end{tabular}

* NIRS = near infrared spectroscopy; SPECT = single-photon emission computed tomography; TCD = transcranial Doppler ultrasonography; Xe-CT = xenon-inhalation computed tomography. 
verse events related to the use of SCS in patients with probable cerebral ischemia in any of these studies (Table 1). The available data on the use of SCS in cerebral ischemia in humans does not support the suggestion that there are significant ill effects associated with it. The efficacy of such an intervention is difficult to gauge, because the published studies are not designed to address this issue. Nevertheless, these studies, combined with the animal data and clinical experience with SCS for pain, provide a compelling reason for the investigation of the feasibility and utility of this intervention.

In this study we have examined the therapeutic limits of SCS as an intervention for the treatment of acute cerebral ischemia. Specifically, we have examined the ability of SCS to reduce stroke volumes when applied after the ischemic insult. Because the clinical application of SCS would necessarily require that it be applied at some point after the onset of ischemia, this is a central issue in the applicability of this technology to patient care. Because current therapeutic interventions for acute stroke have been shown to be safe and effective for up to 3 hours after ischemia only, ${ }^{1}$ in this study we chose to examine the effect of SCS when it was delayed for longer periods.

\section{Materials and Methods}

\section{Animal Preparation}

All experimental protocols were approved by the University of Michigan Committee on the Use and Care of Animals. Adult male Sprague-Dawley rats, each weighing between 250 and $350 \mathrm{~g}$, were selected for the experimental series. The animals were housed in standard conditions in a laboratory environment with free access to food and water.
General anesthesia was induced with 5\% isoflurane (Aerrane). After intubation and initiation of mechanical ventilation with a rodent ventilator (model 683; Harvard Apparatus, Inc.), isoflurane was titrated between 1.5 and $2.25 \%$ to maintain a mean arterial pressure between 80 and $120 \mathrm{~mm} \mathrm{Hg}$ and a normal $\mathrm{PaCO}_{2}$ level between 35 and $45 \mathrm{~mm} \mathrm{Hg}$. The rats were paralyzed with $10 \mathrm{mg} / \mathrm{kg}$ gallamine (Sigma Chemical Co.) given intravenously as a muscle relaxant. The animal's body temperature was maintained at $37^{\circ} \mathrm{C}$ with an automatic heating device (model 73A-YSI; Yellow Springs Instruments). The femoral artery was cannulated for continuous monitoring of arterial blood pressure and arterial blood gas levels.

\section{Occlusion of the MCA}

We used the transcranial MCA occlusion model described by Tamura et al..$^{32}$ to induce permanent focal ischemia in the distribution of the MCA. An incision was made over the temporal aspect of the skull, and a craniectomy was fashioned with a bur drill. The zygomatic arch was also divided to provide adequate visualization. The dura mater was opened directly over the MCA, and the artery was coagulated with the aid of a bipolar electrocoagulator down to $1 \mathrm{~mm}$ below the level of the olfactory tract. The artery was then divided. The incision was closed, and the animal underwent placement of the spinal cord stimulator lead.

\section{Spinal Cord Stimulation}

The animal was placed in a stereotactic frame (model 900; Kopf Instruments). A midline incision was made from the occiput to the cervical spine. The first and second laminae were identified and cleared of paraspinous mus-

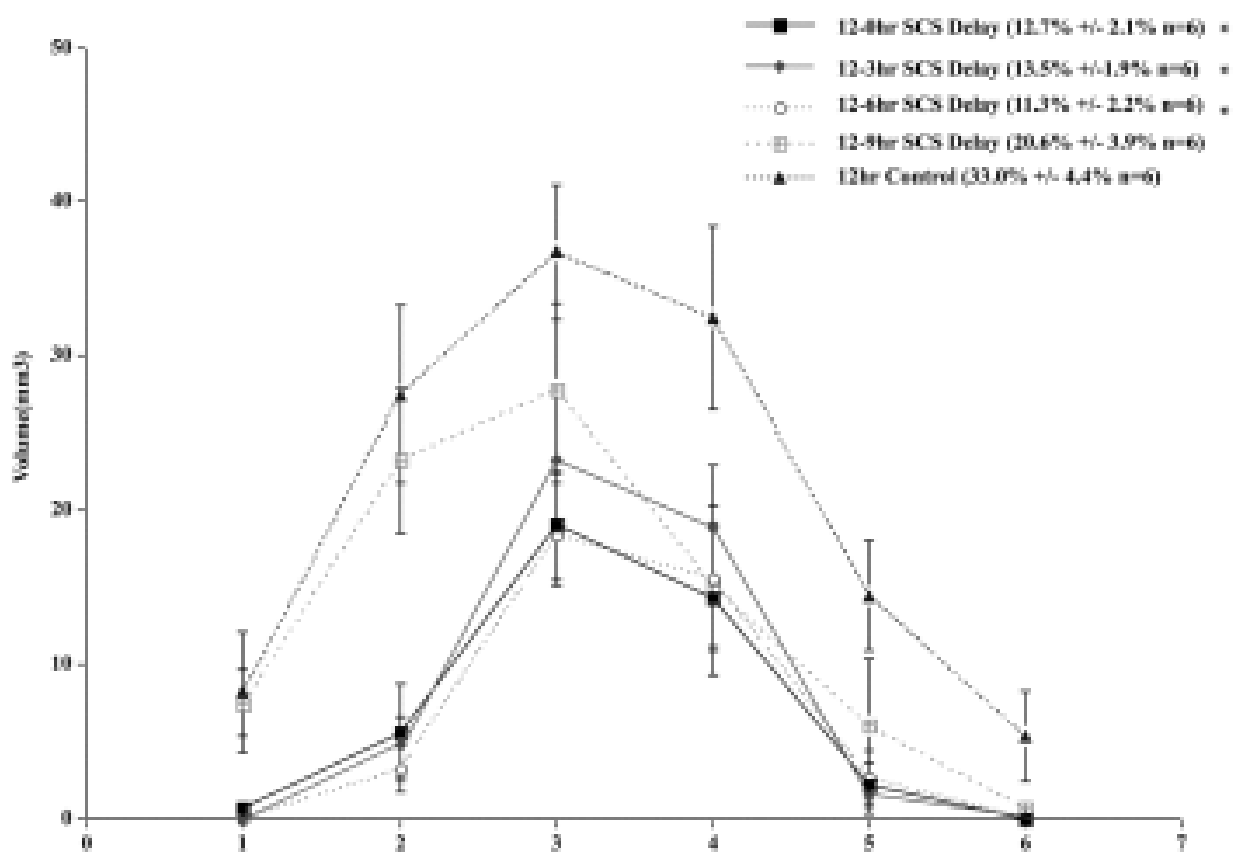

FIG. 1. Summary graph showing that delayed application of SCS reduces stroke volume. The regional slice analysis of TTC staining in the brains of animals undergoing MCA occlusion and SCS is shown. The X-axis indicates the brain section. Areas of infarction were measured in serial sections obtained at 2-mm intervals through the forebrain. Total infarction volumes are also shown for the different groups. $* \mathrm{p}<0.01$ compared with control group. 


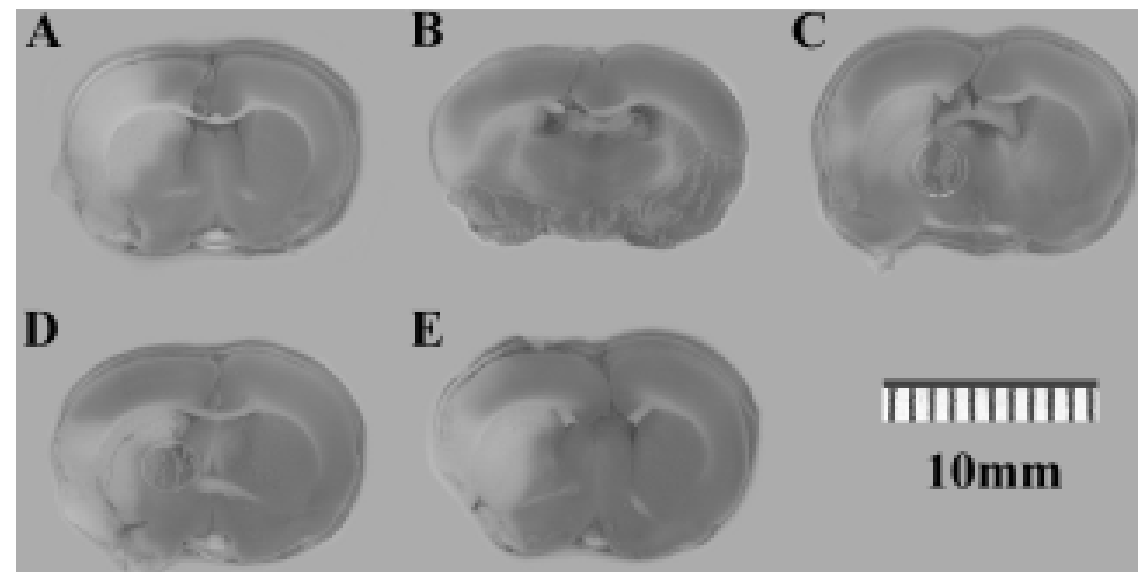

FIG. 2. Coronal sections showing TTC staining of rat brains in the various treatment groups: control (A); immediate SCS (B); 3-hour delay before SCS (C); 6-hour delay before SCS (D); and 9-hour delay before SCS (E). There appears to be a preferential protection of the cortical mantle conferred by SCS.

cles. The interspace between $\mathrm{C}-1$ and $\mathrm{C}-2$ was cleared, sparing the dura mater. A 1-mm platinum ball electrode was placed on the dura and secured in place. A ground electrode was placed in the soft tissues of the neck and the incision was closed. The wires were then brought out through the skin and connected to a standard stimulation setup, which consisted of a stimulator and a constant-current unit (models S48 and CCU1-A, respectively; Grass Instruments). Animals were awakened and allowed free access to food and water for the duration of the experiment. For the next 12 hours the rats were either stimulated immediately or in a progressively delayed fashion (starting at 3,6, or 9 hours after MCA occlusion). Animals were stimulated with a square-wave pulse at an amplitude of $1.5 \mathrm{~mA}$, frequency of $50 \mathrm{~Hz}$, and pulse width of 250 msec. Two-minute stimulation trains were repeated every 10 minutes. These parameters were chosen based on optimal settings derived in previous studies conducted in our laboratory. ${ }^{26,27}$ A control group underwent insertion of the stimulator lead but received no stimulation throughout the experimental period. Animals were killed after the 12hour postoperative period.

\section{Analysis of Stroke Size}

We assessed infarct size by staining the brain with the mitochondrial stain TTC (Sigma Chemical Co.) The brain was cut coronally at 2-mm intervals, and individual slices were soaked for 10 minutes in a solution of $2 \%$ TTC in 0.1 $\mathrm{M}$ phosphate-buffered saline ( $\mathrm{pH} 7.4)$ in a $37^{\circ} \mathrm{C}$ bath. Excess TTC was drained and the slices were refrigerated in a $10 \%$ formalin solution. Images were acquired by placing the brain sections on a color flatbed scanner that was connected to a computer running with image-analysis software (version 1.61; National Institutes of Health). Unstained regions were measured, and the percentage of the total slice area was calculated for each slice to attain a percentage of stroke volume.

\section{Results}

Our previous work has shown that SCS improves CBF in a model of focal ischemia in rats. ${ }^{26}$ In the experimental series presented here we have extended this finding in an attempt to define the limits of such neuroprotection. We found that rats undergoing no stimulation had a 33\% infarction of their hemisphere, as expected. Rats in which stimulation was started at periods as late as 6 hours after occlusion of the MCA experienced significantly smaller infarctions (11.3-13.5\%). Even animals undergoing stimulation as late as 9 hours after ischemia seemed to have smaller infarction volumes, although this did not reach statistical significance (20.6\% compared with $33 \%$ in the 9 -hour compared with control group, $p>0.05$ ). These findings are presented graphically in Fig. 1. Representative sections from TTC-stained brains obtained in the different treatment groups are shown in Fig. 2.

Rats undergoing stimulation for the period of the experiment appeared to tolerate the treatment well. They exhibited no signs of discomfort when the stimulator was on.

\section{Discussion}

Electrical stimulation of the nervous system represents a unique opportunity to influence neural activity and physiology. The use of electrical stimulation in the treatment of pain is the prototype of this type of therapeutic intervention, but the number of applications of stimulation has grown significantly in the past several years. Electrical neurostimulation is now being used to treat movement disorders, myocardial ischemia, and psychiatric disorders. As the technology for delivering electrical impulses in the nervous system improves, our ability to expand the capabilities of this modality continues to advance. As a case in point, the use of SCS to improve CBF appears to extend the window of therapeutic efficacy in an experimental model. A full 6 hours after occlusion of the MCA, SCS appears to confer significant protection from infarction. We are currently investigating the length of time it is necessary to deliver electrical stimulation after the onset of ischemia. It is our hope that a stimulator lead placed percutaneously while the patient is in the emergency department will allow clinicians to ameliorate acute stroke in individuals who are not candidates for thrombolytic therapy.

References 
1. Anonymous: Tissue plasminogen activator for acute ischemic stroke. The National Institute of Neurological Disorders and Stroke rt-PA Stroke Study Group. New Engl J Med 333: 1581-1587, 1995

2. Barolat G: Experience with 509 plate electrodes implanted epidurally from C1 to L1. Stereotact Funct Neurosurg 61: 60-79, 1993

3. Broseta J, García-March G, Sánchez-Ledesma MJ, Goncalves J, Silva I, Barcia JA, et al: High-cervical spinal cord electrical stimulation in brain low perfusion syndromes: experimental basis and preliminary clinical report. Stereotact Funct Neurosurg 62:171-178, 1994

4. Campbell JN: Examination of possible mechanisms by which stimulation of the spinal cord in man relieves pain. Appl Neurophysiol 44:181-186, 1981

5. Cook AW, Oygar A, Baggenstos P, Pacheco S, Kleriga E: Vascular disease of extremities. Electric stimulation of spinal cord and posterior roots. N Y State J Med 76:366-368, 1976

6. Ekre O, Eliasson T, Norrsell H, Wahrborg P, Mannheimer C: Long-term effects of spinal cord stimulation and coronary artery bypass grafting on quality of life and survival in the ESBY study. Eur Heart J 23:1938-1945, 2002

7. Garcia-March G, Sanchez-Ledesma MJ, Anaya J, Broseta J: Cerebral and carotid haemodynamic changes following cervical spinal cord stimulation. An experimental study. Acta Neurochir Suppl 46:102-104, 1989

8. Holsheimer J, Struijk JJ, Rijkhoff NJ: Contact combinations in epidural spinal cord stimulation. A comparison by computer modeling. Stereotact Funct Neurosurg 56:220-233, 1991

9. Holsheimer J, Struijk JJ, Tas NR: Effects of electrode geometry and combination on nerve fiber selectivity in spinal cord stimulation. Med Biol Eng Comput 33:676-682, 1995

10. Holsheimer J, Wesselink WA: Effect of anode-cathode configuration on paresthesia coverage in spinal cord stimulation. Neurosurgery 41:654-660, 1997

11. Hosobuchi Y: Electrical stimulation of the cervical spinal cord increases cerebral blood flow in humans. Appl Neurophysiol 48:372-376, 1985

12. Hosobuchi Y: Treatment of cerebral ischemia with electrical stimulation of the cervical spinal cord. Pacing Clin Electrophysiol 14:122-126, 1991

13. Isono M, Kaga A, Fujiki M, Mori T, Hori S: Effect of spinal cord stimulation on cerebral blood flow in cats. Stereotact Funct Neurosurg 64:40-46, 1995

14. Jivegard LE, Augustinsson LE, Holm J, Risberg J, Ortenwall P: Effects of spinal cord stimulation (SCS) in patients with inoperable severe lower limb ischaemia: a prospective randomised controlled study. Eur J Vasc Endovasc Surg 9:421-425, 1995

15. Kumar K, Toth C, Nath RK, Verma AK, Burgess JJ: Improvement of limb circulation in peripheral vascular disease using epidural spinal cord stimulation: a prospective study. J Neurosurg 86:662-669, 1997

16. Linderoth B, Fedorcsak I, Meyerson BA: Peripheral vasodilatation after spinal cord stimulation: animal studies of putative effector mechanisms. Neurosurgery 28:187-195, 1991

17. Linderoth B, Gunasekera L, Meyerson BA: Effects of sympathectomy on skin and muscle microcirculation during dorsal column stimulation: animal studies. Neurosurgery 29: 874-879, 1991

18. Linderoth B, Herregodts P, Meyerson BA: Sympathetic mediation of peripheral vasodilation induced by spinal cord stimulation: animal studies of the role of cholinergic and adrenergic receptor subtypes. Neurosurgery 35:711-719, 1994

19. Mannheimer C, Eliasson T, Augustinsson LE, Blomstrand C, Emanuelsson $\mathrm{H}$, Larsson $\mathrm{S}$, et al: Electrical stimulation versus coronary artery bypass surgery in severe angina pectoris: the ESBY study. Circulation 97:1157-1163, 1998
20. McNab D, Khan SN, Sharples LD, Ryan JY, Freeman C, Caine $\mathrm{N}$, et al: An open label, single-centre, randomized trial of spinal cord stimulation vs. percutaneous myocardial laser revascularization in patients with refractory angina pectoris: the SPiRiT trial. Eur Heart J 27:1048-1053, 2006

21. Meglio M, Cioni B, Visocchi M, Nobili F, Rodriguez G, Rosadini G, et al: Spinal cord stimulation and cerebral haemodynamics. Acta Neurochir (Wien) 111:43-48, 1991

22. Melzack R, Wall PD: Pain mechanisms: a new theory. Science 150:971-979, 1965

23. Myklebust JB, Cusick JF, Boerboom LE, Prieto TE, Khan TA: Vascular effects of spinal cord stimulation in the monkey. Stereotact Funct Neurosurg 64:32-39, 1995

24. North RB: Spinal cord stimulation for intractable pain: longterm follow-up. J Spinal Disord 3:356-361, 1990

25. Patel S, Huang DL, Sagher O: Sympathetic mechanisms in cerebral blood flow alterations induced by spinal cord stimulation. J Neurosurg 99:754-761, 2003

26. Sagher O, Huang DL: Effects of cervical spinal cord stimulation on cerebral blood flow in the rat. J Neurosurg 93 (1 Suppl): $71-76,2000$

27. Sagher O, Huang DL, Keep RF: Spinal cord stimulation reducing infarct volume in a model of focal cerebral ischemia in rats. J Neurosurg 99:131-137, 2003

28. Shealy CN, Mortimer JT, Reswick JB: Electrical inhibition of pain by stimulation of the dorsal columns: preliminary clinical report. Anesth Analg 46:489-491, 1967

29. Shinonaga M, Takanashi Y: Vasodilating effect of spinal cord stimulation for cerebral vasospasm. Acta Neurochir Suppl 77:229-230, 2001

30. Stanton-Hicks M, Salamon J: Stimulation of the central and peripheral nervous system for the control of pain. J Clin Neurophys 14:46-62, 1997

31. Takanashi Y, Shinonaga M: Spinal cord stimulation for cerebral vasospasm as prophylaxis. Neurol Med Chir (Tokyo) 40: 352-357, 2000

32. Tamura A, Graham DI, McCulloch J, Teasdale GM: Focal cerebral ischaemia in the rat: 1 . Description of technique and early neuropathological consequences following middle cerebral artery occlusion. J Cereb Blood Flow Metab 1:53-60, 1981

33. Ubbink DT, Vermeulen H: Spinal cord stimulation for critical leg ischemia: a review of effectiveness and optimal patient selection. J Pain Symptom Manage 31 (4 Suppl): S30-S35, 2006

34. Visocchi M, Cioni B, Pentimalli L, Meglio M: Increase of cerebral blood flow and improvement of brain motor control following spinal cord stimulation in ischemic spastic hemiparesis. Stereotact Funct Neurosurg 62:103-107, 1994

35. Visocchi M, Cioni B, Vergari S, Marano G, Pentimalli L, Meglio M: Spinal cord stimulation and cerebral blood flow: an experimental study. Stereotact Funct Neurosurg 62:186-190, 1994

36. Visocchi M, Giordano A, Calcagni M, Cioni B, Di Rocco F, Meglio M, et al: Spinal cord stimulation and cerebral blood flow in stroke: personal experience. Stereotact Funct Neurosurg 76:262-268, 2001

Manuscript received October 3, 2006.

Accepted in final form October 25, 2006.

This work was supported by National Institutes of Health Grant No. R01-nS41061.

Address reprint requests to: Oren Sagher, M.D., Department of Neurosurgery, University of Michigan Health System, 1500 East Medical Center Drive, Ann Arbor, Michigan 48109. email: osagher@umich.edu. 